\title{
The Effects of Network Loads and Latency in Multiplayer Online Games*
}

\author{
Jin Ryong Kim, Il Kyu Park, and Kwang Hyun Shim \\ Digital Content Research Division \\ Electronics and Telecommunications Research Institution \\ Daejeon, Republic of Korea \\ \{jessekim,xiao, shimkh\} @etri.re.kr
}

\begin{abstract}
In this work, we performed variety of tests to see the effects of network loads and latency in multiplayer online games. We applied hierarchical load testing architecture using large scale virtual clients to find out the bottlenecks of the game servers. We also investigated the effect of latency on multiplayer online games. We controlled network game packets (i.e. packet delay, packet drop rate, packet duplication rate, packet reordering rate) to see the impact on real-time multiplayer games.
\end{abstract}

Keywords: Load tests, multiplayer games, MMOG tests, P2P-based tests, network game tests, mean opinion score.

\section{Introduction}

Recent technologies in broadband network and 3D rendering have made it possible to provide high quality and large scaled 3D online games. Advances in 3D graphics hardware and rendering software technologies enabled people to enjoy realistic 3D games. The increase in residential broadband Internet connections encouraged the popularity of multiplayer online games.

However, the growth in the popularity of interactive multiplayer network games has increased the importance of a better understanding of the effects of network loads and network latency. In massively multiplayer online games (MMOG), large scales of players enter single virtual world concurrently and interact with each other. MMOG are very large distributed applications and shares very large states. However, one of the main issues in MMOG is how to handle massive amount of network packets. Most of MMOGs have client-server based network architecture. That is, the server has to process all the network and I/O. The overhead of handling all the communication among clients through the server can result in long delays and a large amount of consumed networking resources. Thus, the server can be a single point of failure.

In peer-to-peer multiplayer online games, handling the real-time interaction among peers is one of the main challenging issues. Many games such as first person shooters (FPS), casual sports games, and racing games adopt peer-to-peer architecture along

This work is funded by MIC of Korea and the project number is 2006-S-044-01. 
with client-server architecture. It is because they have high requirements in maintaining the consistency of the virtual world. In this case, the latency is the main issue. Most online games have low bit-rate requirements, sending frequent but small packets. They require prompt response from tens to hundreds msec. They are very sensitive in delayed packets and require very low thresholds in latency depending on the genre of the games.

\section{Related Work}

A lot of research work has been done on testing and analyzing multiplayer online games. Fritsch et al. [1] illustrated the effect of latency and network limitations on MMORPGs. They conducted and analyzed different combinations of user actions using Everquest 2 [2]. Dick et al. [3] measured the effect of latency on performance in multiplayer games using GOS and MOS methodologies. The authors introduced the subject MOS and the objective game outcome score as a normalized metric for multiplayer games. They analyzed that various multiplayer games behave differently under the same network conditions. Pantel et al. [4] analyzed the impact on real-time multiplayer games using car racing simulation. They concluded that a delay of more than $100 \mathrm{~ms}$ should be avoided for a racing game. The authors mentioned that a delay up to $50 \mathrm{~ms}$ is uncritical for a car-racing game. They believed that dead-reckoning has the drawback that the prediction error exponentially increases with increasing network delays. Beigeder et al. [5] illustrated the effects of loss and latency on user performance in Unreal Tournament 2003 [6]. In their work, they discovered that the first person shooting (FPS) game players can notice latencies over $100 \mathrm{~ms}$.

\section{Approach}

\subsection{Load Tests Methodology}

A load testing architecture for MMOG should be able to create hundreds to thousands loads to ensure the stability of the servers. In order to perform load tests on MMOG, we present a hierarchical load testing methodology [7].

Figure 1 illustrates a hierarchical load testing methodology for MMOG. It consists of master host, a number of agent hosts and virtual clients on their agent host. In this methodology, we put one master host to manage a number of agent hosts. The number of agent hosts can be increased constantly in parallel and create as many virtual clients as up to their hardware performance. The virtual clients can connect with servers and create actual loads and they are controlled by a master host via their agent host.

The master host controls its agent hosts and deliver event packets to agent hosts. The master host exists as an application entity and manages overall load test.

One or more agent hosts communicate with a master host. They actually interpret received commands from a master host and manage their virtual clients. The roles of agent hosts are to create, control, remove or monitor their virtual clients. The virtual clients are the replacement of actual client application and they are controlled by a master host. The virtual clients enter the virtual game worlds by connecting to the server. They join the game and interact with each other. 


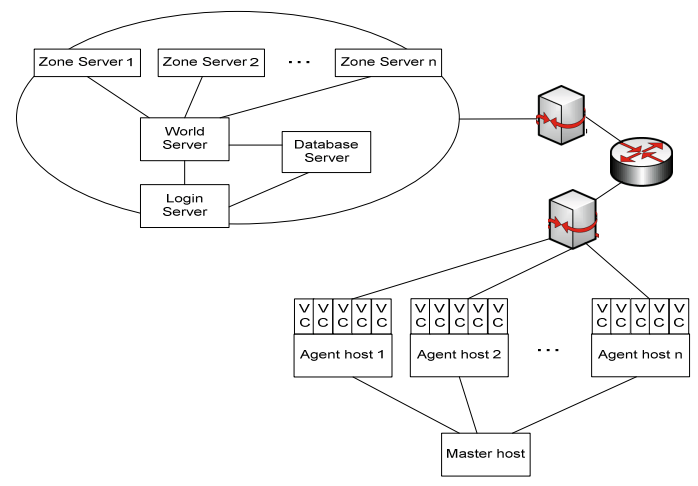

Fig. 1. Hierarchical load testing architecture

\subsection{Peer-to-Peer Based Tests Methodology}

In peer-to-peer network game tests, we set virtual network environment for each client host to get their own network environment. For networking factors, we controlled packet delay, packet drop probability, packet duplication and packet reordering rate. We selected one peer-to-peer based multiplayer online game: online FPS game.

Figure 2 illustrates the network topology for our sample games. The roles of the servers are to manage the client connections, login information, and manage the game channels and game rooms. Once the game players get together in a game room, each game player connects up with other peers. Within the peers, one becomes the master host (server) and others become slave hosts (clients). This game application uses TCP/IP for the client-server mode and UDP/IP for the peer-to-peer mode.

To find out the effect of network latency in multiplayer online games, we used the Mean Opinion Score (MOS). The MOS is the arithmetic mean of all the individual

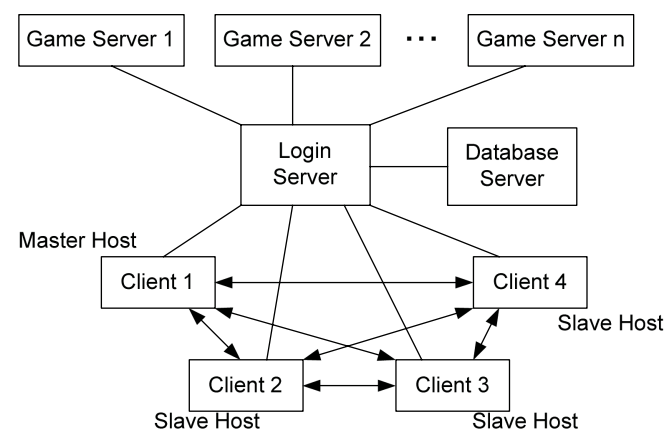

Fig. 2. Hybrid network topology in typical peer-to-peer network games 
Table 1. The Mean Score Opinion Values

\begin{tabular}{lll}
\hline MOS & Quality & Impairment \\
\hline 5 & Excellent & No noticeable impairments \\
4 & Good & Minor impairment noticeable \\
3 & Fair & Clearly impaired environment \\
2 & Poor & Very annoying environment \\
1 & Bad & Unacceptable environment \\
\hline
\end{tabular}

scores, and can range from 1 (worst) to 5 (best). The MOS provides a numerical indication of the perceived quality of received media after compression and/or transmission. In our case, we used the MOS values in [3]. The MOS values for peer-to-peer based tests are illustrated in Table 1.

\section{Experiment}

\subsection{MMOG Load Tests}

The purpose of this test is to find out the bottleneck point when the large number of virtual clients communicates with the server in the same time. In this experiment, we set the virtual clients to send 1 Kbye packets to the server in every one second. The number of virtual clients increased from 500 to 3,000 and the location of the virtual clients were widely distributed in the virtual world. We measured round trip time (delay) for each case.

Figure 3 is the results of this test. In this test, the round trip delay is constantly increased when the number of virtual clients is increased. The memory of the server remained stable but the network and CPU usage has been increased constantly.
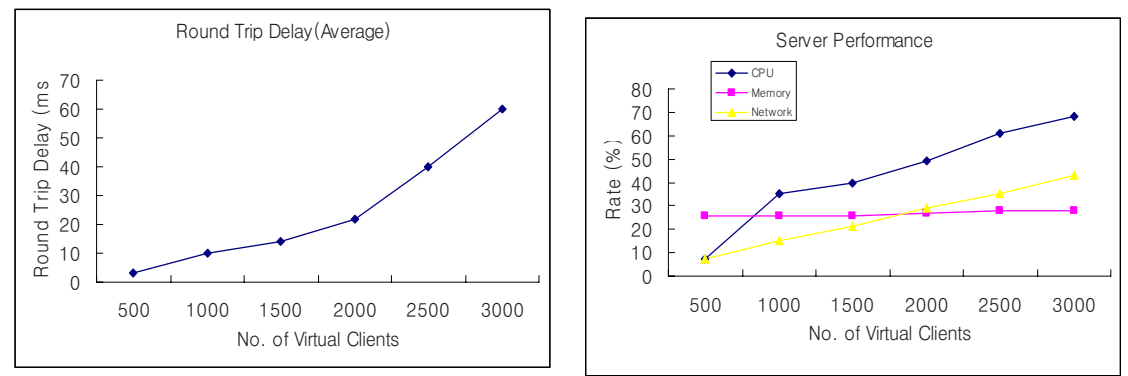

Fig. 3. Round trip delay and server performance for large scale packet transmission test

\subsection{Peer-to-Peer Network Environment Tests}

The purpose of this test is to find out the bottleneck point when we controlled the network environment factors. As illustrated in Figure 7, we set two scenarios: (a) controlling network packets in master client, (b) controlling network packets in slave client. 


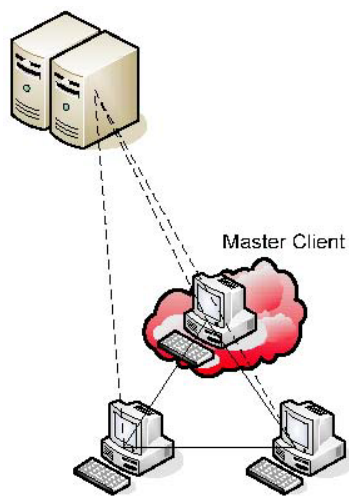

(a)

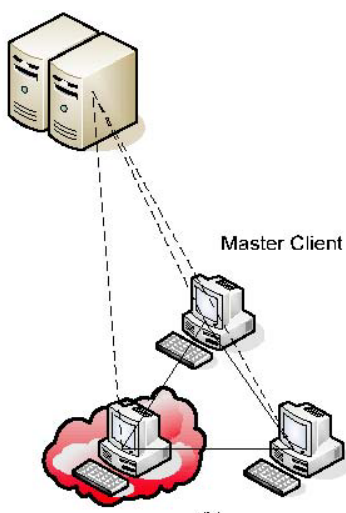

(b)

Fig. 4. Hybrid network topology for peer-to-peer based multiplayer games. (a) Scenario 1, (b) Scenario 2
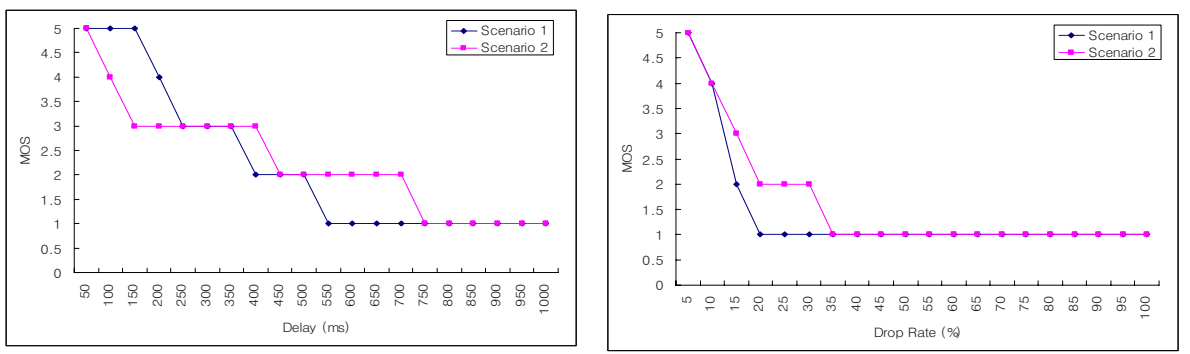

Fig. 5. Packet delay and packet drop tests for scenario 1 and 2

Figure 5 is the result of packet delay and drop tests. In this figure, we noticed that the game players can play without noticeable impairments when the delay is under 200 msec for scenario 1 and under $100 \mathrm{msec}$ for scenario 2 . We also found out that the game players can notice the impairments when the packet drop rate is over $10 \%$.

We also carried out the packet duplication test, but the game content was not affected at all.

\section{Conclusion}

In this work, we performed variety of tests on both MMOG and peer-to-peer based multiplayer online games to find out the effects of network loads and network latency. We used a hierarchical load testing architecture to test the performance of the servers in MMOG. We also carried out peer-to-peer based tests by changing network factors (packet delay, packet drop rate, and packet duplication). For this test, we used MOS values. In results, we noticed that packet delay of $100 \mathrm{~ms}$ and packet drop rate of $10 \%$ are the critical points for peer-to-peer multiplayer online games, especially FPS games. 


\section{References}

1. Fritsch, T., Ritter, H., Schiller, J.: The effect of latency and network limitations on MMORPGs: A field study of Everquest 2. In: Proceedings of the Fourth ACM Network and System Support for Games (NetGames) Workshop, Hawthorne, NY. ACM Press, New York (October 10-11, 2005)

2. Everquest2: http://www.everquest2.com

3. Dick, M., Wellnitz, O., Wolf, L.: Analysis of factors affecting players performance and perception in multiplayer games. In: Proceedings of the Fourth ACM Network and System Support for Games (NetGames) Workshop, Hawthorne, NY. ACM Press, New York (October 10-11, 2005)

4. Pantel, L., Wolf, L.: On the impact of delay on real-time multiplayer games. In: Proceedings of the ACM International Workshop on Network and Operating System Support for Digital Audio and Video (NOSSDAV), Miami, pp. 23-29. ACM Press, New York (May 2002)

5. Beigbeder, T., Coughlan, R., Lusher, C., Plunkett, J., Agu, E., Claypool, M.: The effects of loss and latency on user performance in Unreal Tournament 2003. In: Proceedings of ACM Network and System Support for Games Workshop (NetGames), Portland, OR, pp. 144-151. ACM Press, New York (September 2004)

6. Unreal Tournament (2003), http://www.unrealtournament.com/ut2003

7. Lim, B., Kim, J., Shim, K.: Hierarchical load testing architecture using large scale virtual clients. In: Proceedings of IEEE International Conference on Multimedia and Expo 2006 (ICME 2006), Toronto, Ontario, Canada (July 9-12, 2006) 